\title{
VISCOELASTIC WEB CURL DUE TO STORAGE IN WOUND ROLLS
}

\author{
By \\ S. Pan, A. Azoug and J. K. Good \\ Oklahoma State University \\ USA
}

\begin{abstract}
While bending strains result from any web being wound at a radius of curvature into a roll, these bending strains are largest for the thicker homogeneous webs and laminates. Many webs are viscoelastic on some time scale and bending stresses will lead to creep. When the web material is unwound and cut into discrete samples, a residual curvature will remain. This curvature, called curl, is the inability for the web to lie flat at no tension. Curl is an undesirable web defect that causes loss of productivity in a subsequent web process. The goal of this research is to develop numerical and experimental tools by which process engineers can explore and mitigate machine direction curl in homogenous webs. Two numerical methods that allow the prediction of curl in a web are developed, a winding software based on bending recovery theory and the implementation of dynamic simulations of winding. One experimental method directly measures the curl online by taking advantage of the anticlastic bending resulting from the curl. All methods applied to a common isotropic LDPE web correlate well with each other and present an opportunity for process engineers to mitigate curl and its negative consequences at low time cost.
\end{abstract}

\section{INTRODUCTION}

The mission of winding models was to (1) predict the elastic residual stresses in rolls that resulted from winding $[1,2]$ and $(2)$ use these stresses to predict and mitigate damage of the web in the roll. Webs are often considered viscoelastic because these residual stresses can either decrease or increase markedly in the time and environment that wound rolls remain in storage $[3,4]$. Web damage resulting from winding and storage has been identified qualitatively by categorizing the damage into various types of wound roll defects. Smith [5] developed such an anthology of web defects. Each defect is described followed by known causes and remedies.

Curl is defined as the inability of a web to lie flat when laid upon a flat surface.

There is more than one type of web curl (Fig. 1). An example of machine direction (MD) curl is shown alongside the corresponding wound roll (Fig. 1(a)). This type of curl is the 
focus of this publication. The cut sample of web in Figure 1(a) resided within the wound roll at a given radius. As a result of winding, residual stresses developed and the web was subjected to a combination of MD membrane and bending stresses. Since most webs are viscoelastic on some time scale, creep phenomena are expected as a result of these stresses. Creep will occur faster in time at the outside of a layer, where the combined bending and membrane stress is largest, than at the inside of the layer, where the combined MD stresses will be smallest. This difference in length change is the source of the observed MD curl.

MD curl is often visible as cross machine direction (CMD) curl when unwinding. The web tension in the unwind zone will draw the MD curl flat but because of anticlastic bending [6] and Poisson's ratio of the web material the web will curl in the CMD direction as shown in Figure 1(b). This CMD curl can deter web quality if the magnitude is sufficient to cause contact and potential scratching with elements in the web process line such as the air support drying bars shown in Figure 1(b). A more complex form of curl that has both MD and CMD components is shown in Figure 1(c). This form of curl is not attacked in this publication but demonstrates a case where MD and CMD residual stresses in the lower surface of the web are greater than those in the upper surface.

Web laminates can also exhibit MD curl, with multiple sources of the defect. Usually laminates are strain-matched at the laminator to prevent or reduce MD curl. Laminated webs are often thick and the bending stresses that result from storage at a particular radius in a wound roll can make these viscoelastic webs susceptible to MD curl as a result of roll storage.

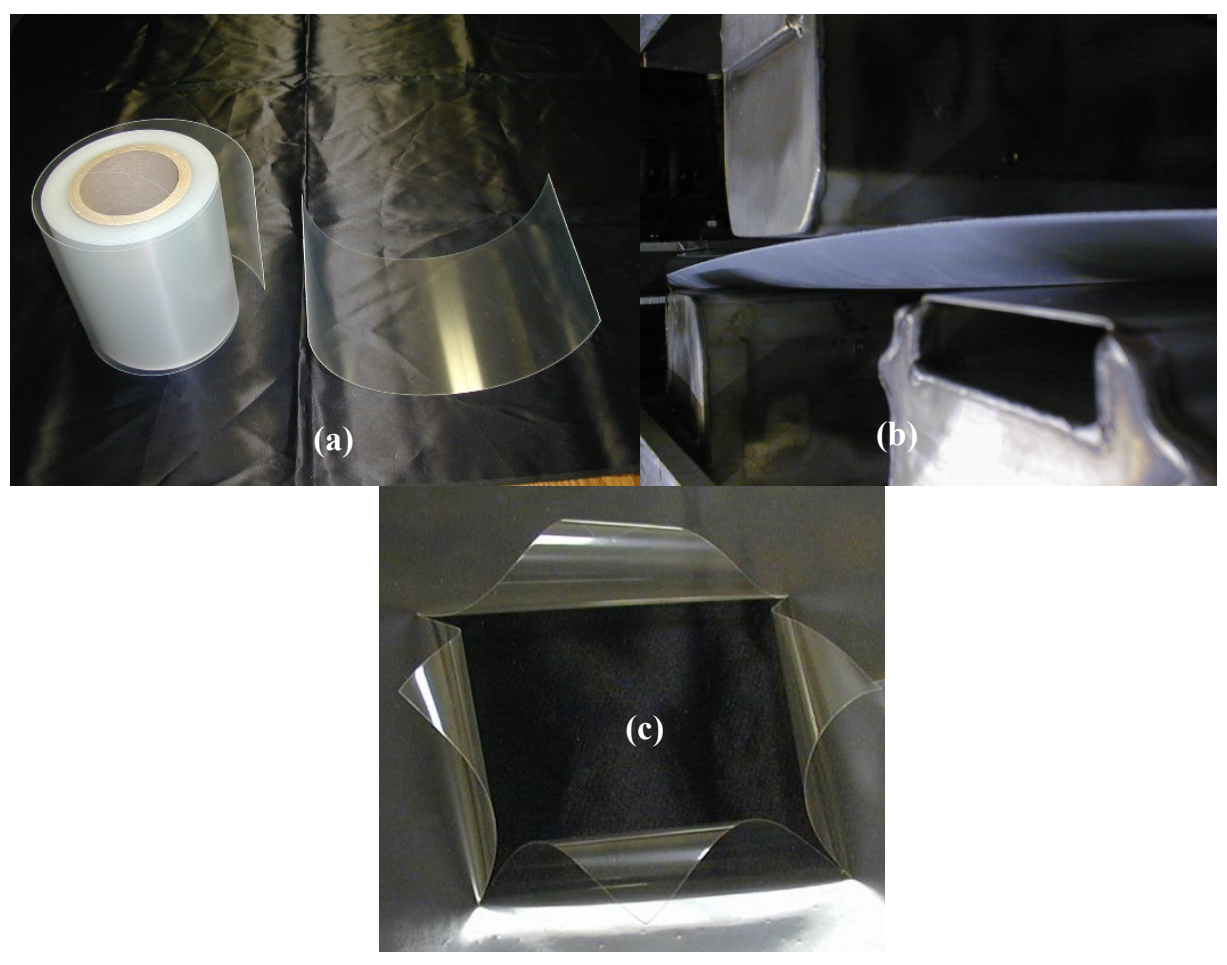

Figure 1 - (a) an example of MD curl, (b) CMD curl and (c) complex curl. 
The patent literature provides some references to sources and mitigation of MD curl. Mitigation often involves reversed winding where the inside of a layer at the unwind becomes the outside of that layer at the rewind. Reverse winding is often done in conjunction with elevated storage temperatures in between reversed windings. [7-9].

From a scientific perspective, when a flat viscoelastic film is forced to conform to a fixed radius of curvature for a period of time, the radius of curvature increases instantaneously to a finite value upon release and further recovers with time. Given enough time the web would completely recover and return to the flat state. This kind of experiment shows that the curl is a reversible phenomenon due to time-dependent effects in the viscoelastic material.

Greener [10] developed and verified experimentally a theoretical expression to predict the curl. His long-term storage analysis then showed that the aging effect in polymer materials may significantly influence core-set curl in polymeric film, unless the storage time is relatively short compared with the age or life of the web [11]. Kidane [12] presented a 2D elastic curl model that was based on laminate theory in 2009.

In order to solve and benchmark curl problems, measurement of the curl is necessary. Swanson developed a curl measurement instrument called the Kappa Gauge [13]. This curl gage supports beams of webs such that the deformation due to gravity is minimized. The instrument also allows measurement of curl in an up or down configuration. Averaging the results for both configurations further reduces the error due to gravity. The Kappa Gage has output units of curvature $(1 / \mathrm{m})$ which is the inverse of the radius of curvature. This is a destructive measurement method; web coupons must be cut such that that the curl curvature can be measured.

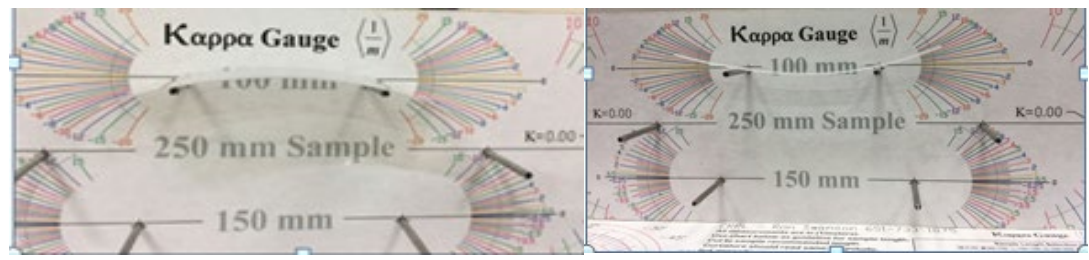

Figure 2 - The Swanson Kappa Gage

\section{DEVELOPMENT}

\section{Bending Recovery Theory}

The Greener model [10] predicts the values of bending recovery (BR). The MD curl that results from a winding process is similar to the bending recovery effect described by Greener. The bending recovery is the portion of an imposed bending deformation that is recovered with time by viscoelastic relaxation. Greener defined the bending recovery $B R$ as:

$$
B R=\frac{R}{\rho}
$$

where $\mathrm{R}$ is the radius at which the web layer was wound into the roll and $\rho$ is the radius of curvature of a web coupon free of web stress or tension cut from that same web layer immediately after unwinding. Many factors may influence the curl, including the radius the web layer was wound into the roll, the thickness of the web, the viscoelastic properties of the web, the length of storage time, the environmental conditions, the winding stresses, etc. If the web is flat after unwinding, then per expression $\{1\}$ the BR 
would be 0 . If the BR is equal to unity, the web is totally viscoelastic and there is no elastic recovery.

Greener developed a phenomenological model for the bending recovery in equation $\{2\}$, based on a simple extension of the classic bending theory for a linear viscoelastic material.

$$
B R=\left[1-\frac{E_{t}\left(t_{r}\right)+E_{c}\left(t_{r}\right)}{2 E_{0}}\right]
$$

where $t_{r}$ is the storage time, $E_{0}$ is the initial instantaneous modulus, that is the instantaneous modulus at time 0 when the web was deformed from the flat state to a radius $\mathrm{R}, E_{t}(t)$ is the relaxation modulus in tension, and $E_{c}(t)$ the relaxation modulus in compression. The modulus $E_{0}$ should be measured in conditions where there is no viscoelastic stress relaxation. This may include testing below the glass transition temperature or at high strain rate. Greener measured $E_{0}$ via uniaxial tensile tests at a strain rate of $100 \% / \mathrm{min}$ at $21^{\circ} \mathrm{C}$. The relaxation processes in the tensile and compressive zones of bending stress can be dissimilar, which makes the problem more complex [14]. For some polymers the compressive stresses relax substantially slower than the tensile stresses. In that case, $E_{t}$ and $E_{c}$ would take different values.

The bending recovery can be related to time and viscoelastic properties rather than the tension or the radius of the web layer. Measurement of the compressive relaxation modulus is difficult in thin webs, and two extreme situations will be considered.

When the compressive stresses relax substantially slower than tensile stresses, and thus $E_{c}\left(t_{r}\right)=E_{0}$ :

$$
B R_{1}=\frac{1}{2}\left[1-\frac{E_{t}\left(t_{r}\right)}{E_{0}}\right]
$$

When the stress relaxation of the stresses is independent of the sign of the stress, then $E_{c}\left(t_{r}\right)=E_{t}\left(t_{r}\right)$ :

$$
B R_{2}=1-\frac{E_{t}\left(t_{r}\right)}{E_{0}}
$$

The simplest curl calculations for webs wound into rolls would result from ignoring the effects of winding membrane residual stresses entirely. The curl radius of a web after a period of time $t_{r}$ in storage in a wound roll would be calculated using either expression $\{3\}$ or $\{4\}$ and knowledge of the radius $\mathrm{R}$ at which the layer was wound into the roll.

\section{Extension of a Winding Code for Curl Analysis}

The developed code for predicting curl allows the use of any of the bending recoveries expressed by equations $\{2-4\}$. This code assumes that a viscoelastic web without any initial curl was wound into a roll and stored for a time period, perhaps at elevated temperature. The user desires to know what curl would be present after unwinding and relieving any web tension. A flow chart of the code is shown in Figure 3.

In the code developed herein, the winding model has an embedded assumption that the winding occurs so quickly that the process is entirely elastic. The larger storage time, however, provides ample time for stress relaxation and creep due to the viscoelastic properties of the web. Depending on how the routine blocks are written, the mechanical viscoelastic behavior of the web is equivalently represented by relaxation or creep functions. The viscoelastic code, which follows that of Qualls $[3,4]$, requires orthotropic creep function $J(t)$. 
Measurements of $J_{r}(t)$ were conducted in stack tests in which the stack was only subject to compression stress (i.e. radial pressure in the wound roll). The stack would cease to be a continuum if attempts were made to subject it to tension normal to the web layers, because of layer separation. Thus, characterization of $J_{r}(t)$ only in compression is reasonable. Measurements of $J_{\theta(t)}$ were only conducted in tension. These tests were conducted by subjecting strips of web cut with their long axis in the MD to constant MD stress using dead weights. Qualls assumed the creep functions that couple radial stress to circumferential creep $J_{r} \theta(t)$ and circumferential stress to radial creep $J_{\theta_{r}}(t)$ were negligible.

Neither Qualls, nor any other developers of viscoelastic wound roll codes, considered how the sign of stress could affect the creep functions. The output of winding codes demonstrates the circumferential stresses are typically high near the core and the outside of the wound roll but in the interior of the roll these stresses can near zero or become negative. The degree to which they can become negative depends on the level of winding tension, the relative values of the elastic modulus in the circumferential direction $E_{\theta}$ and the state-dependent radial modulus $E_{r}\left(E_{r}=K_{2}\left(P(r)+K_{l}\right)\right.$ where $K_{l}$ and $K_{2}$ are Pfeiffer's constants [15] and $P(r)$ is the radial pressure dependent on radius), the core stiffness and the core and final wound roll radius. If $J_{\theta}(t)$ depends on the sign of the stress, this could be a shortcoming of current viscoelastic wound roll codes depending on how different $J_{\theta_{t}}(t)$ and $J_{\theta_{c}}(t)$ are.

Finally, Greener's curl analysis [11] requires the input of relaxation functions, both in compression and tension. Based on the viscoelastic characterization performed by the user, conversion of creep functions to relaxation functions or vice versa can be required to satisfy the needs of the different analysis codes. This conversion is the focus of the first block in the code after reading the input (Fig. 3). The conversion procedure from Baumgaertel and Winter [16] was implemented.

A decision block then reviews whether the relaxation functions in tension and compression are equal.

- If they are equal, then the membrane stresses calculated by the winding code and the viscoelastic boundary value code are not required and the decision block bypasses those codes and proceeds to computations of bending recovery directly using expression $\{4\}$.

- If the relaxation modulus in tension and compression differ now the membrane stresses combined with the bending stresses will determine what portion of the thickness of the web will be subject to a tensile stress and what portion will be subject to a compressive stress. Now the winding and viscoelastic boundary value codes must be executed to obtain the membrane stresses through time and the bending recovery is determined using expression $\{2\}$.

The validation example will assume the relaxation modulus in tension is equal to that in compression. To calculate the curl radius, equation $\{4\}$ will be used:

$$
\rho(R)=\frac{R}{B R_{2}}=\frac{R}{\left[1-\frac{E_{t}\left(t_{r}\right)}{E_{0}}\right]}
$$

where $\rho_{(r)}$ is the recovered radius of curvature for a web stored at a radius $R$ for a time period $t_{r}$ in a wound roll. 


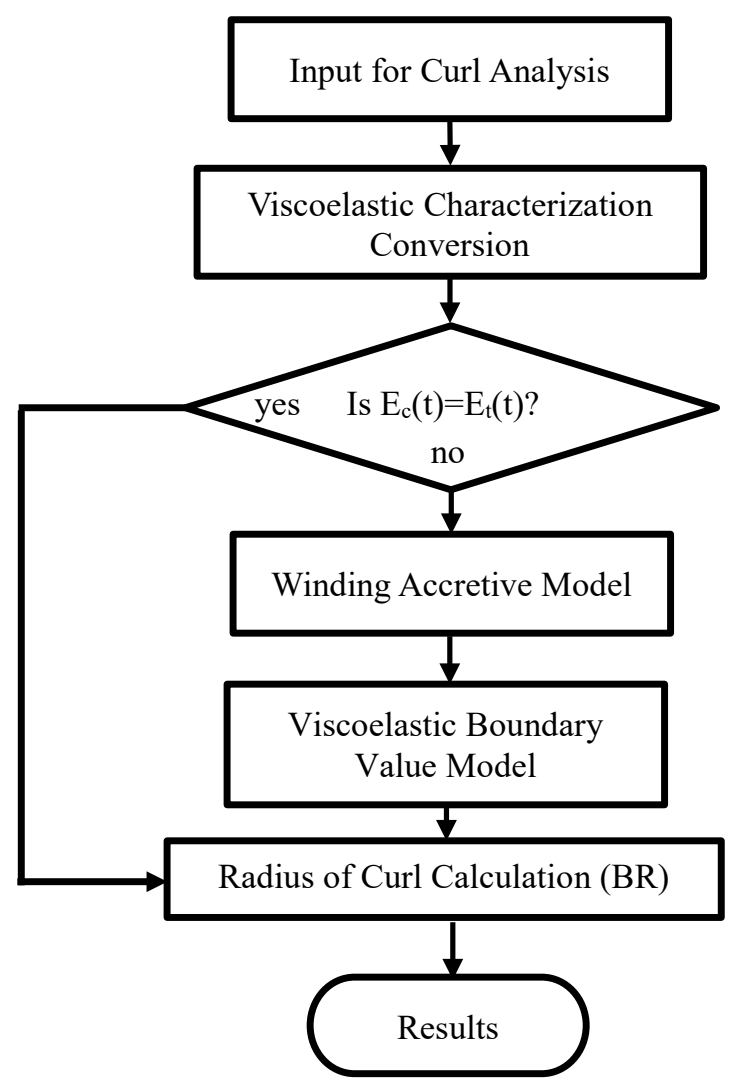

Figure 3 - Flow Chart of Curl Analysis Code

Validation: A low-density polyethylene (LDPE) web $10.2 \mathrm{~cm}$ (4 in) wide and 0.508 $\mathrm{mm}$ (0.02 in) thick was used to verify the curl analysis model. The instantaneous modulus $E_{0}$ of LDPE is the inverse of the instantaneous compliance $J_{0}, E_{0}=1 / J_{0}=153 \mathrm{MPa}$, which was measured at a strain rate of $10 \% / \mathrm{min}$. Creep tests were performed using different dead weights $(44.5,66.7,89 \mathrm{~N})$ to exert different stress levels on web samples. The instantaneous strain, corresponding to the stress multiplied by the instantaneous modulus, was subtracted from the total measured strain leaving the creep strain through time. The creep strains were normalized by dividing by the applied stress levels. These normalized creep strains condensed to essentially one curve, from which it can be inferred that the LDPE film tested is a linear viscoelastic material. The creep function was then obtained by fitting a 2-term Prony series to the normalized creep data:

$$
J(t)=J_{0}+\sum_{i=1}^{2} J_{i}\left(1-\exp \left(-t / \tau_{i}\right)\right)
$$

where $J_{i}$ are compliances and $\tau_{i}$ are the retardation times. The LDPE elastic and viscoelastic properties are provided in Table 1 . 


\begin{tabular}{|c|c|c|c|c|}
\hline$J_{0}(1 / \mathrm{KPa})$ & $J_{1}(1 / \mathrm{KPa})$ & $\tau_{1}(\mathrm{~s})$ & $J_{2}(1 / \mathrm{KPa})$ & $\tau_{2}(\mathrm{~s})$ \\
\hline $6.53 \mathrm{E}-06$ & $2.90 \mathrm{E}-06$ & 1913 & $1.16 \mathrm{E}-06$ & 100,566 \\
\hline
\end{tabular}

Table 1 - Creep Function for LDPE film at $21^{\circ} \mathrm{C}$

Using expressions $\{4\}$ and $\{5\}$ requires knowledge of the relaxation function, which will be expressed as a Prony series for convenience:

$$
E(t)=E_{0}-\sum_{i=1}^{n} E_{i}\left(1-\exp \left(-t / \lambda_{i}\right)\right)
$$

where $E_{i}$ are the relaxation moduli and $\lambda_{i}$ are the relaxation times. The creep and relaxation functions are related by the convolution:

$$
\int_{0}^{t} E(t) J(t-\zeta) d \zeta=t
$$

Analytically converting a creep function to the relaxation function is cumbersome. The conversion method [15] allows to convert discrete spectra efficiently. Applying that method, the relaxation times $\lambda_{i}$ will be the roots equation $\{9\}$.

$$
\begin{gathered}
\left(\prod_{i=1}^{m}\left(s \lambda_{i}+1\right)\right)\left(\prod_{i=1}^{m}\left(s \tau_{i}+1\right)\right)= \\
\left(\sum_{i=0}^{m} J_{i} \prod_{j \neq i}^{m}\left(s \lambda_{j}+1\right)\right)\left(\sum_{i=0}^{m} E_{i} \prod_{j \neq i}^{m}\left(s \tau_{j}+1\right)\right)
\end{gathered}
$$

The relaxation modulus terms $E_{i}$ are expressed in equation $\{10\}$ :

$$
E_{i}=E_{0} \frac{\prod_{j=1}^{m}\left(\frac{\tau_{i}}{\lambda_{j}}-1\right)}{\prod_{j \neq i}^{m}\left(\frac{\tau_{i}}{\tau_{j}}-1\right)}
$$

The relaxation moduli and times for this LDPE film per equation $\{7\}$ are shown in Table 2:

\begin{tabular}{|c|c|c|c|c|}
\hline$E_{0}(\mathrm{MPa})$ & $E_{1}(\mathrm{MPa})$ & $\lambda_{1}(\mathrm{~s})$ & $E_{2}(\mathrm{MPa})$ & $\lambda_{2}(\mathrm{~s})$ \\
\hline 153.09 & 47.17 & 1,325 & 11.45 & 89,630 \\
\hline
\end{tabular}

Table 2 - Relaxation Modulus for LDPE film at $21^{\circ} \mathrm{C}$

The winding curl tests were conducted as follows:

1. The LDPE web was conditioned by reversed winding to remove as much curl due to previous storage as possible. The web was unwound from a roll and the outside of the outer layer would become the inside of the layer that was wound on to a downstream winder. In between reversed windings, the roll was stored at $65^{\circ} \mathrm{C}$ for 5 hours. Sample coupons of the inner and outermost layers were harvested, followed by measurements of MD curl using the Kappa Gage. When both MD curls were less than 2 units on the Kappa Gage (or a curl radius less than $0.5 \mathrm{~m}$ ), the web was assumed to be essentially flat and ready for further testing. 
2. The web was rewound at the designated winding tension and stored at room temperature $\left(21^{\circ} \mathrm{C}\right)$ for 1 or 3 day(s).

3. The curl of a sample coupon harvested from the outer layer was measured on the Kappa Gage.

4. The roll was then unwound so that a web coupon adjacent to the core could be harvested. The curl of the innermost layer was measured.

5. This procedure was repeated for various winding tensions.

All the input parameters for the winding and storage simulations are provided in the Appendix. However, since we assume $E_{t}\left(t_{r}\right)=E_{c}\left(t_{r}\right)$, these simulations will have no impact on the curl obtained with the model. It is however informative to understand how the wound roll pressures and tangential stresses are changing through time. In this case, the winding tension was $40 \mathrm{~N}$, which exerts a $775 \mathrm{KPa}$ membrane stress on the web. In Figure 4, both the membrane stress and the combined membrane and bending stress on the inner and outer surface of the web can be reviewed. The majority of the stress the web witnesses is tensile through the thickness and the use of the tensile relaxation function in Table 2 is reasonable. The model curl in Kappa units was determined using the inverse of the curl radius in equation $\{5\}$ (Fig. 5).

The comparison of the experimental and modeling results for the MD curl are presented in Table 3 . The results from the model, which can be calculated directly from equation $\{5\}$ in this case, agree with the measured test values quite well. The absolute error is less than the benchmark of 2 used to establish whether the web conditioned for these tests was essentially flat prior to winding and storage. To improve the agreement between equation $\{5\}$ and tests further would first require better methods for preconditioning the web to ensure the entire web length was curl free prior to tests.
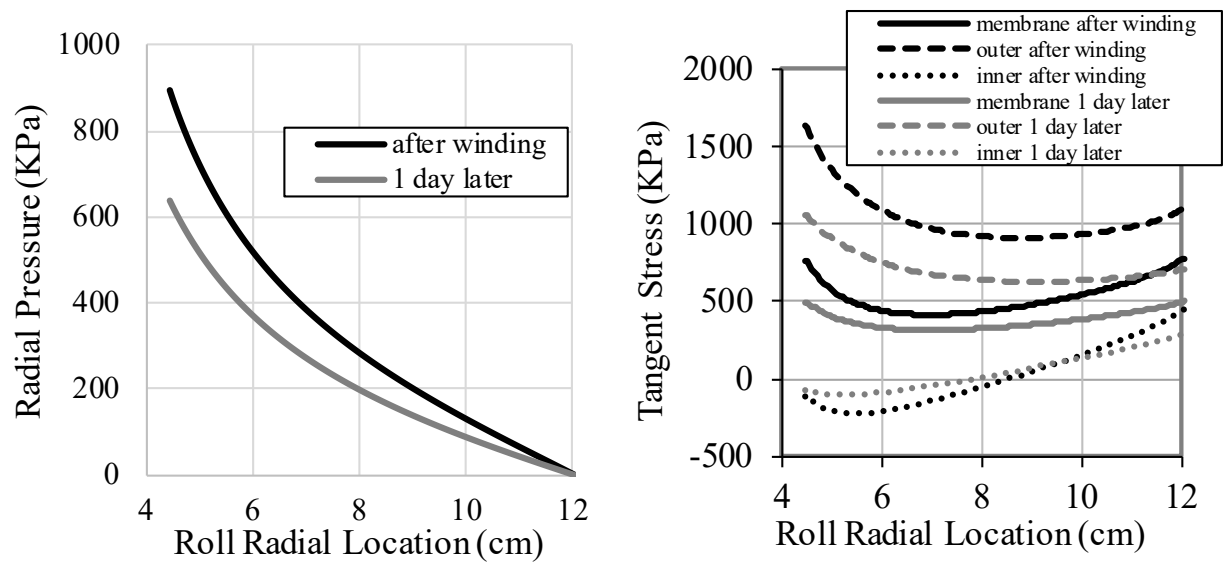

Figure 4 - The pressure and tangential stress due to winding and storage of the LDPE web at a winding tension of $40 \mathrm{~N}$, corresponding to case 1 in Table 3 . 


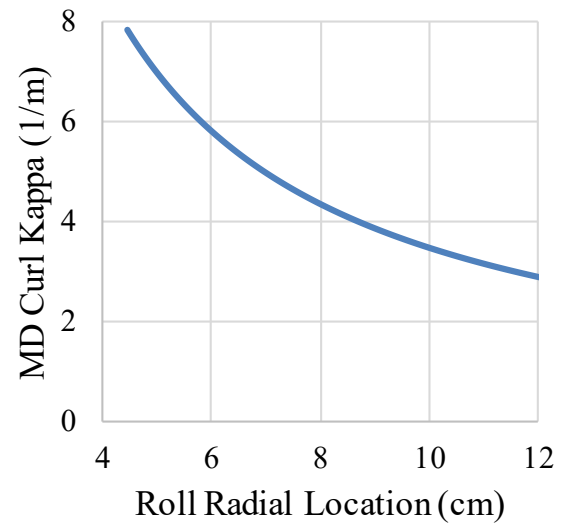

Figure 5 - The MD Curl due to winding and storage of the LDPE web in Table 3.

\begin{tabular}{|c|c|c|c|c|c|c|c|c|c|c|}
\hline \multirow[t]{2}{*}{ Test } & \multirow[t]{2}{*}{$\begin{array}{c}\mathrm{T} \\
(\mathrm{N})\end{array}$} & \multirow[t]{2}{*}{$\begin{array}{l}\text { Temp } \\
\left({ }^{\circ} \mathrm{C}\right)\end{array}$} & \multirow{2}{*}{$\begin{array}{l}\text { Core } \\
\text { Radius } \\
(\mathrm{cm})\end{array}$} & \multirow{2}{*}{$\begin{array}{l}\text { Outer } \\
\text { Radius } \\
(\mathrm{cm})\end{array}$} & \multicolumn{3}{|c|}{$\begin{array}{c}\text { Innermost Layer } \\
\text { (Kappa 1/m) }\end{array}$} & \multicolumn{3}{|c|}{$\begin{array}{c}\text { Outermost } \\
\text { (Kappa 1/m) }\end{array}$} \\
\hline & & & & & Measurec & Model & |error| & Measured & Model & |error| \\
\hline 1 & \multirow{3}{*}{40} & \multirow{5}{*}{21} & 4.45 & 12.1 & 6.5 & 7.7 & 1.2 & 2.5 & 2.9 & 0.4 \\
\hline 2 & & & 4.45 & 11.4 & 9.0 & 7.7 & 1.3 & 3.5 & 3.1 & 0.4 \\
\hline 3 & & & 4.45 & 11.0 & 8.5 & 7.7 & 0.8 & 2.5 & 3.2 & 0.7 \\
\hline 4 & 80 & & 4.45 & 10.9 & 8.5 & 7.7 & 0.8 & 2.5 & 3.2 & 0.7 \\
\hline 5 & 22 & & 4.45 & 10.9 & 9.0 & 7.7 & 1.3 & 2.5 & 3.2 & 0.7 \\
\hline
\end{tabular}

Table 3 - Comparison between Lab Tests and Model Results for LDPE $\left(21^{\circ} \mathrm{C}\right)$

\section{Curl Analysis using Dynamic Simulation Codes}

Another method for simulating MD curl due to viscoelastic creep is through dynamic simulations. Abaqus/ $\mathrm{CAE}^{1}$ is a deformation-based finite element code and uses the relaxation function to model the viscoelastic behavior of materials. Abaqus allows the user to input isotropic viscoelastic parameters in built-in constitutive models. However, Qualls [3,4] demonstrated the viscoelastic behavior of web in wound rolls was better modeled as orthotropic. Non-isotropic viscoelastic behavior can only be implemented through user-written subroutines that update the behavior after each time step in the dynamic simulation. These subroutines (UMAT for standard and VUMAT for explicit solutions) are required even for simulation of elastic winding due to the state-dependent radial modulus $E_{r}(P)$ previously discussed. Since we will only wind one layer, the material can be considered isotropic without affecting the solution. It also greatly simplifies the simulations. For more complex winding simulations, a dedicated anisotropic UMAT should be developed.

There are many sets of parameters that can define the viscoelastic behavior of a material in Abaqus. The user can provide shear test data (stress relaxation, creep, or sinusoidal oscillations), or the user can directly enter the shear moduli and time constants of a representative Prony series for the relaxation or creep function. In this study, the shear relaxation moduli and time constants, as well as the bulk modulus were the selected

${ }^{1}$ Dassault Systems, Abaqus Simulia, Rising Sun Mills, 166 Valley St., Providence, RI 02909-2499 
form of input. As shear tests are not easily conducted on thin webs, we performed tensile stress relaxation tests (Table 2) and converted the tensile relaxation function to a shear relaxation function. Assuming the measured relaxation function (Table 2) represents the isotropic behavior of the web, and the Poisson's ratio $v$ is time-independent, the shear relaxation modulus can be derived from equation $\{7\}$ :

$$
G(t)=\frac{E(t)}{2(1+v)}=\frac{E_{0}}{2(1+v)}-\sum_{i=1}^{n} \frac{E_{i}}{2(1+v)}\left(1-e^{\left(-\frac{t}{\tau_{i}}\right)}\right)
$$

The shear relaxation modulus would have the following Prony series form:

$$
G(t)=G_{0}-\sum_{i=1}^{n} G_{i}\left(1-e^{\left(-\frac{t}{\tau_{i}}\right)}\right)
$$

The time-dependent terms $G_{i}$ are then normalized by the instantaneous shear modulus $G_{0}$ :

$$
g_{i}=\frac{G_{i}}{G_{0}}=\frac{\frac{E_{i}}{2(1+v)}}{\frac{E_{0}}{2(1+v)}}=\frac{E_{i}}{E_{0}}
$$

The normalized bulk modulus $K(t)=E(t) /[3(1-2 v)]$ Prony terms are similarly determined and become numerically equivalent to the normalized shear relaxation modulus terms:

$$
k_{i}=\frac{K_{i}}{K_{0}}=\frac{\frac{E_{i}}{3(1-2 v)}}{\frac{E_{0}}{3(1-2 v)}}=\frac{E_{i}}{E_{0}}
$$

The simulation parameters for the viscoelastic behavior of the LDPE are shown in Table 4.

\begin{tabular}{|c|c|c|c|}
\hline \multicolumn{4}{|c|}{ Linear, Isotropic, Prony Series Definition } \\
\hline $\mathrm{i}$ & $g_{i}$ & $k_{i}$ & $\tau_{i}(\mathrm{~s})$ \\
\hline 1 & 0.308 & 0.308 & 1,325 \\
\hline 2 & 0.075 & 0.075 & 89,630 \\
\hline
\end{tabular}

Table 4 - Viscoelastic parameters for the LDPE web, input in the simulation.

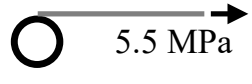

Step 1 - Pretension, core constrained

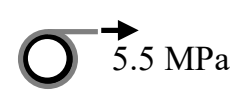

Step 2 - Winding core turns $1 \mathrm{rev} \mathrm{ccw}$

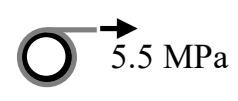

Step 3 - Storage

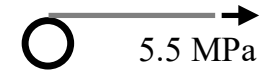

Step 4 - Unwinding core turns $1 \mathrm{rev} \mathrm{cw}$

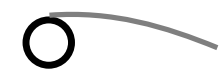

Steps $5 \& 6$ - Release MD curl forms

Figure 6 - Dynamic Simulation Steps. $(\mathrm{cw}=$ clockwise, $\mathrm{ccw}=$ counterclockwise $)$

The simulation wound the same LDPE film described in the validation section of the winding code $(10.2 \mathrm{~cm}$ wide and $0.0508 \mathrm{~cm}$ thick). The core was defined as an analytical rigid surface with a $1.27 \mathrm{~cm}$ radius. The web was modeled with CPS4R solid elements and the Abaqus Standard solution procedure was used. The isotropic elastic part of the behavior of the web was characterized by a Young's modulus of $153 \mathrm{MPa}$ and a Poisson's ratio of 0.3 . The isotropic viscoelastic part was characterized by the parameters 
in Table 4. The simulation is organized in six phases: pretension, winding, storage, unwinding, release, final curl (Figure 6 and Table 5). The duration of each step of this dynamic simulation critically controls the influence of the dynamic behavior on the simulation results.

As the web is released in Step 5, dynamic effects lead to large movements of the web. During step 6, the web reaches a static equilibrium in a stress-free curled state, i.e. the final state. The curl radius is assessed in the last 3 seconds of the simulation using the deformed coordinates of the web. Figure 7 shows the final deformed state of the web that was stored for 1 day, unwound, and released. The deformed coordinates of each node were probed along the MD direction. Three consecutive nodes were used to define an arc and compute the arc radius. Inverting the radius of the arc yields the curvature of the center node. The bending recovery corresponds to the curvature divided by the radius of the core.

\begin{tabular}{|l|c|c|}
\hline Step Name & Time & Step Description \\
\hline 1 Pretension & $1(\mathrm{~s})$ & 5.5 MPa tension applied to the right surface of web \\
\hline 2 Winding & $1.25(\mathrm{~s})$ & 1 lap of web wound on the core \\
\hline 3 Storage & 1 or 3 days & 2 different storage times set up \\
\hline 4 Unwinding & $1.25(\mathrm{~s})$ & Opposite rotation of the winding process \\
\hline 5 Release & $0.1(\mathrm{~s})$ & Winding tension was released very quickly \\
\hline 6 Final State & $100(\mathrm{~s})$ & $\begin{array}{c}\text { Allow dynamic effects to decay and make estimate curl } \\
\text { prior to creep decay }\end{array}$ \\
\hline
\end{tabular}

Table 5 - Step Description of Curl Simulation

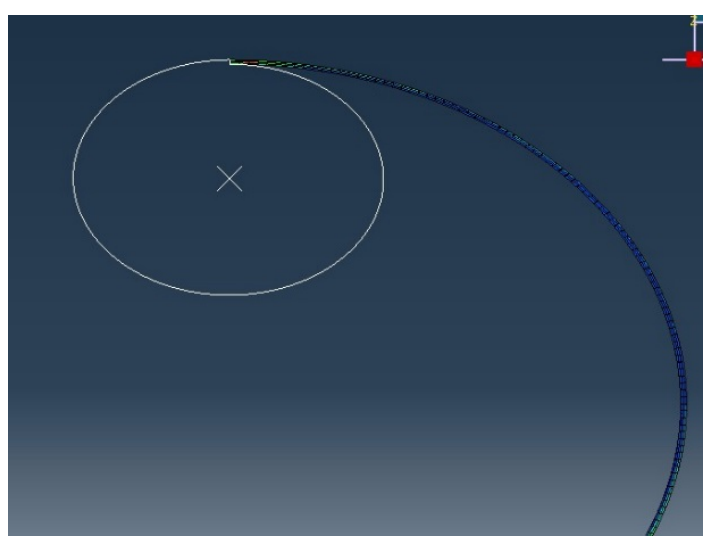

Figure 7 -Web geometry after 1day of storage, unwinding, and tension release

The results are presented in Figures 8 and 9 for one and three days of storage. The dynamic simulations report a significant bending recovery, which implies a significant curl in the web after 1 and 3 days of storage. Since the largest time constant is $89,630 \mathrm{~s}$ (1.04 days), this considerable curl was expected. Also shown are the bending recovery predicted by the bending recovery theory using BR1 (equation $\{3\}$ ) and BR2 (equation $\{4\}$ ). The prediction from BR2 (equation $\{4\}$ ) agrees best with the dynamic simulation results (Figs 8 and 9). As the constitutive model does not differentiate between tension 
and compression and is fully isotropic, $\mathrm{BR} 2$, where $E_{c}\left(t_{r}\right)=E_{t}\left(t_{r}\right)$, logically corresponds to the behavior of the simulated web.

The bending recovery did not evolve appreciably during 2 more days of storage. The largest time constant in the relaxation function being 89,630 seconds or 1.05 days, a large part of the time-dependent behavior is already relaxed after one day. Because larger time constants are not considered in this constitutive model, additional creep or changes in bending recovery would not be predicted by the simulations or the theory.

In conclusion, we showed that simulations of curl within a wound roll are possible using commercial dynamic simulation codes like Abaqus. The code can also predict the decrease in curl with time after unwinding depending on the time constants in the Prony series of the relaxation function. Simulation of cases where $E_{c}\left(t_{r}\right) \neq E_{t}\left(t_{r}\right)$ would be possible as the properties of the finite elements can be partitioned through the thickness. The simulation would be computing the combined membrane and bending stresses, which can cause the neutral axis to shift resulting in the partition of the compressive and tensile stresses varying.

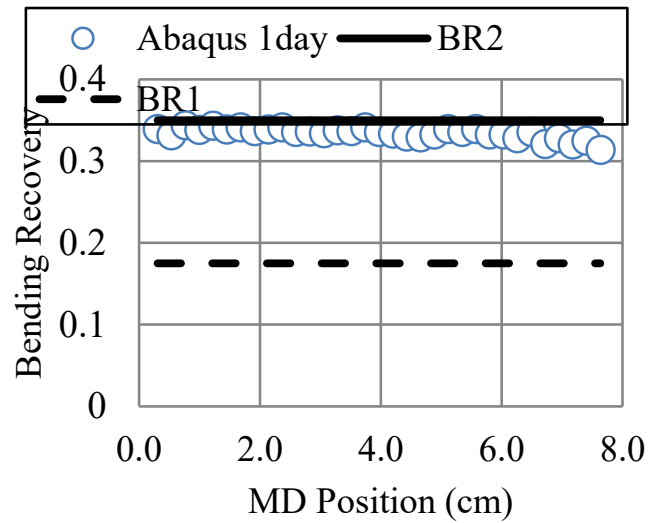

Figure 8 - Bending recovery theory versus dynamic simulation results, 1 day of storage.

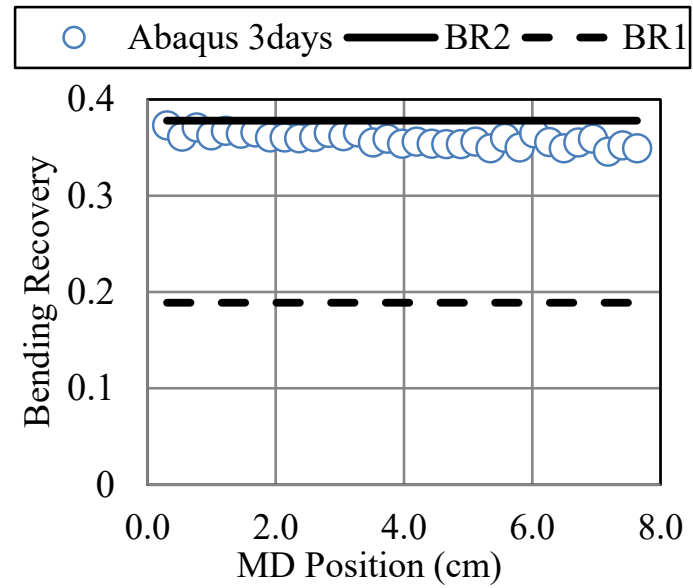

Figure 9 - Bending recovery theory versus dynamic simulation results, 3 days of storage. 


\section{ONLINE MEASUREMENT OF MD CURL}

\section{Theory of anticlastic bending}

From Euler beam theory, the bending moment $M$ that would be required to make a web of thickness $h=0.508 \mathrm{~mm}$, width $w=10.2 \mathrm{~cm}$, modulus $E=153 \mathrm{MPa}$ and Poisson ratio $v=0.3$ to conform to a cylindrical roller of radius $r=4.45 \mathrm{~cm}$ would be:

$$
\mathrm{M}=\frac{E I}{\mathrm{r}}=3.82 \mathrm{~N}-\mathrm{mm}
$$

The parameters chosen here are identical to those of the web used previously in the validation of the theory and in the simulations. The radius of the core is identical as well. If this moment was applied to MD edges of a web coupon as a distributed moment $\left(M_{x}=M / w\right)$, a shape more complex than that of a cylinder would be witnessed, a saddle shape (Figure 10). The coupon has a radius of curvature $r$ in the MD but a curvature in the CMD is also witnessed. This is due to the web plate being a three-dimensional structure. The edge moments induced tensile bending strains on the outside surface of the web and compressive strains on the inside surface. The Poisson effect coupled these MD strains to CMD strains $\left(\varepsilon_{\mathrm{MD}}=-v \varepsilon_{\mathrm{CMD}}\right)$. This results in negative CMD strains on the outer surface of the web and positive CMD on the inner surface. The deformed shape of the web is well-known as an anticlastic surface [6].

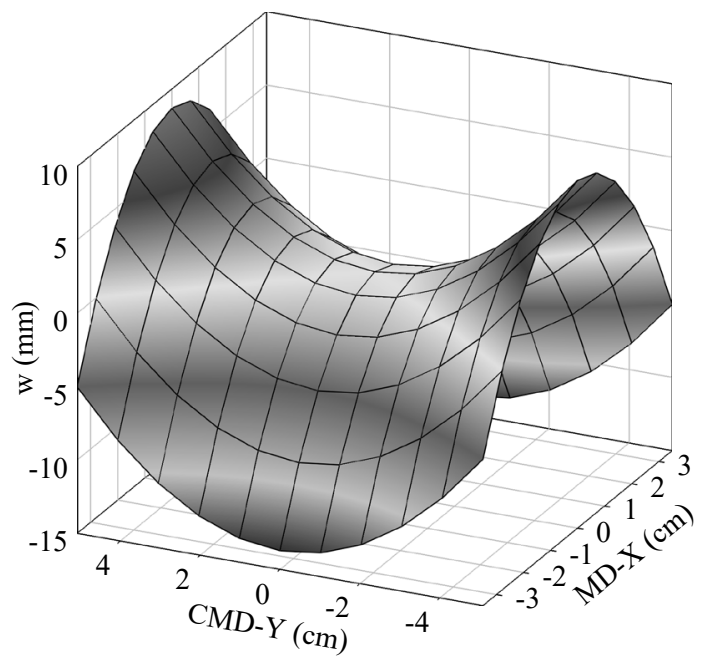

Figure 10 - The LDPE deforming to an anticlastic surface.

The web can be forced to conform to a cylindrical surface only if there is a second bending moment, $M_{y}=v M_{x}$, applied on the CMD edges. The equation of the surface deformation $w$ seen in Figure 10 is:

$$
w=-\frac{M_{x}}{2 D\left(1-v^{2}\right)} x^{2}+\frac{v M_{x}}{2 D\left(1-v^{2}\right)} y^{2}
$$

where $D$ is the plate bending stiffness $\left(E h^{3} /\left(12\left(1-v^{2}\right)\right)\right)$ and $x$ and $y$ are MD and CMD coordinates whose origin are central to the web coupon in Figure 10. If the Poisson's ratio vanished, there would be no variation in deformation in the CMD and the web 
would take the deformed shape of a cylinder of radius $r$. Although the Poisson's ratio is generally not zero, anticlastic deformations are not witnessed when a web is wound into a roll. This is due to the out-of-plane forces that the web experiences while winding. Web tension acts to create a contact pressure $P$ beneath the outer layer and all layers beneath witness even higher pressures $[1,2]$ that cause the web to conform to a cylindrical core and remain cylindrical during winding. The CMD strains due to anticlastic behavior remain in the web but, due to viscoelastic relaxation, they diminish through time. When coupons are harvested from a roll for curl measurement, the cylindrical shape in Figure 1 (a) is witnessed.

If a roll of curled web material is unwound into a web line, the web tension would elastically pull out the MD curl of the web, however due to anticlastic bending a CMD curl will be induced. If this CMD curl could be measured and related to the MD curl in the wound roll then the MD curl could be assessed continuously during unwinding. This method is preferable to cutting specimens out since the curl is expected to change throughout a wound roll that has been in storage. Furthermore, if the CMD curl could be assessed with non-contact measurement then curl testing could be accomplished nondestructively. If the web assumed an MD curl of radius $R_{M D}$ while in storage and if the MD curl was drawn flat by tension when unwinding, theoretically the out-of-plane deformation in the CMD would be:

$$
w=\frac{v M_{x}}{2 D\left(1-v^{2}\right)}=\frac{v}{2 R_{M D}\left(1-v^{2}\right)} y^{2}
$$

The $2^{\text {nd }}$ derivative of $w$ is related to the CMD curl radius $R_{C M D}$ :

$$
\frac{\partial^{2} w}{\partial y^{2}}=\frac{v}{R_{M D}\left(1-v^{2}\right)}=\frac{1}{R_{C M D}}
$$

Thus, if the CMD radius of curvature in the free span $R_{C M D}$ can be measured, the MD radius of curl $R_{M D}$ can be inferred:

$$
R_{M D} \approx R_{C M D} \frac{v}{1-v^{2}}
$$

Web tension will not remove all the out-of-plane deformation $w$ in the MD in the free span. This deformation will be affected by web constraints at rollers, web elasticity, web tension, and span length. Thus equation $\{19\}$ is only an estimate of the MD curl.

Several LDPE specimens, $0.508 \mathrm{~mm}$ thick, $10.2 \mathrm{~cm}$ wide and $45 \mathrm{~cm}$ long, were prepared. These specimens were wound and then stored at $65^{\circ} \mathrm{C}$ for 2 hours to remove the initial curl. The MD and CMD curvature was measured with a Kappa Gage (Table 6).

\begin{tabular}{|c|c|c|c|c|c|}
\hline Specimen & 1 & 2 & 3 & 4 & 5 \\
\hline MD Curl Kappa & 2.50 & 2.00 & 1.25 & 4.25 & 1.0 \\
\hline CMD Curl Kappa & 3.00 & 1.00 & 3.75 & 5.25 & 1.0 \\
\hline
\end{tabular}

Table 6 - Initial MD and CMD Curl of Specimen

This proves that both MD and the CMD curl can exist in a stress-free state. Some CMD curl can exist independently from the MD curl and be unrelated to anticlastic bending. Initial CMD unrelated to anticlastic bending could affect the accuracy of an online measurement system based on the correspondence between MD and CMD curl. 


\section{Characterizing the Relationship between MD and CMD Curl}

The out-of-plane web displacement was measured online using a Keyence ${ }^{2}$ LK031 laser displacement sensors. The sensors are mounted $30 \mathrm{~mm}(1.2 \mathrm{in})$ away from the undeformed web plane (Figure 11). The measuring range is $\pm 5 \mathrm{~mm}(+0.2$ in). One sensor is targeted at the CMD center of the web while the other two are targeted symmetrically at $3.8 \mathrm{~cm}$ (1.5 in) away. The length of vertical test span is $45 \mathrm{~cm}$ (18 in). The CMD curl radius was inferred from the measured displacement. The relationship between MD and CMD curl was characterized with the following procedure:

(1) $5 \mathrm{LDPE}$ specimens of $5 \mathrm{~m}(16.4 \mathrm{ft})$ in length were prepared. Their MD curl levels was measured off-line in a stress-free state using the Kappa Gage.

(2) Each specimen was then transported under tension through the test span, shown in Figure 10.

(3) These samples were short and the winding and unwinding were manually assisted in order to attain the target web tension as quickly as possible $(13.3 \mathrm{~N}$, 26.7N, 40.0N).

(4) The 3 averaged signals for out-of-plane deformation measured at the $3 \mathrm{CMD}$ locations $(-3.8,0.0,3.8 \mathrm{~cm})$ were averaged for the length of the web and used to define an arc from which the CMD curl radius was inferred.

(5) Every test was repeated at least 3 times on a web specimen. Finally, a relationship between initial MD curls (no tension) and CMD curl (online under tension) is obtained.

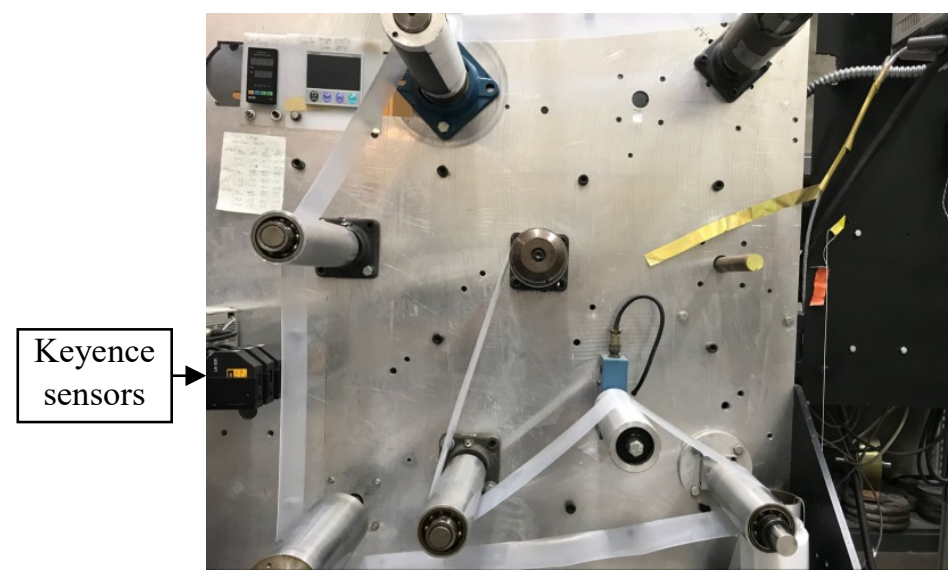

Figure 11 - On-line measurement of CMD Curl

\footnotetext{
${ }^{2}$ Keyence Corporation of America, 500 Park Blvd., Suite 200, Itasca, IL 60143, USA
} 


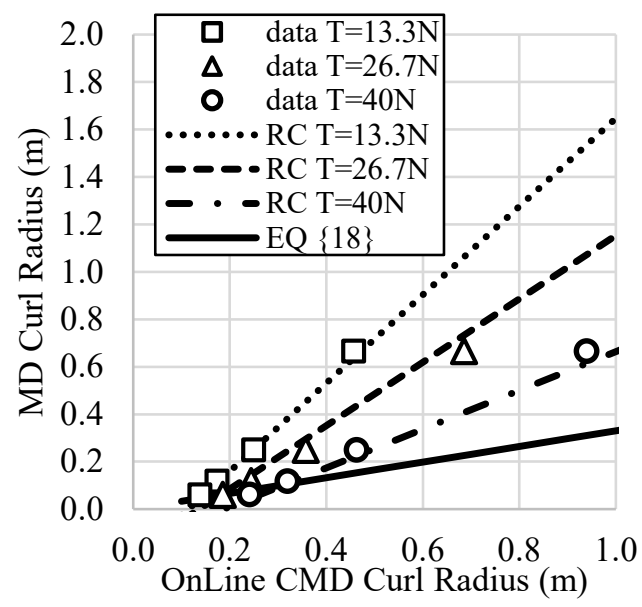

Figure 12 - Relationship between MD Curl and Measured CMD Curl

The experimental results (Fig. 12) demonstrate a linear relationship between MD and CMD curl, as the theory predicted (equation $\{19\}$ ). The data further demonstrates a dependency of the MD-CMD curl linear relationship on web tension whereas equation $\{19\}$ depended only on Poisson's ratio. For this web and test span, a regression curve can be developed to infer the MD curl in a stress-free web state from the measured CMD curl in the web under tension. The linear regression is:

$$
\text { MDcurl }=[-0.0390 T+2.3787] \text { CMDcurl }+[0.0022 T-0.2420]
$$

where the MD and CMD curls are in meters and $T$ is the web tension in Newtons. This regression curve is compared with experimental data for the 3 tension levels (Figure 12). The agreement with the experimental data is good. However, the regression curve developed herein is applicable to a unique test span and web material. Such a regression curve could also be developed using a dynamic simulation.

\section{COMPARISON OF ONLINE MEASUREMENT AND WINDER SOFTWARE CURL ANALYSIS}

In the previously performed validation tests, the MD curl was only measured at the innermost and outermost layers using the Kappa Gage. The standard adopted for wound roll preparation for curl testing was that the initial Kappa measurement should be less than 2 after reversed windings with intermediate storage. The online measurement method was used to explore this initial MD curl, previously considered negligible. An identical LDPE was used. The core radius was $4.45 \mathrm{~cm}$ and the outer radius of the wound rolls was $8.9 \mathrm{~cm}$. 


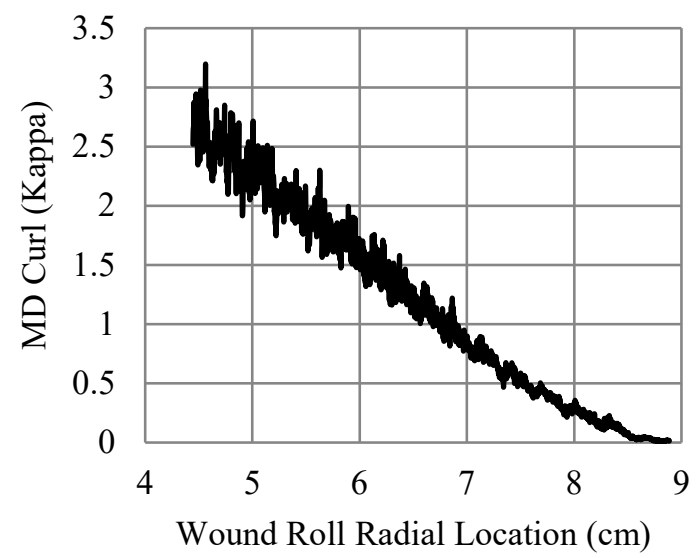

Figure 13 - Online MD Curl after Removing Initial Curl

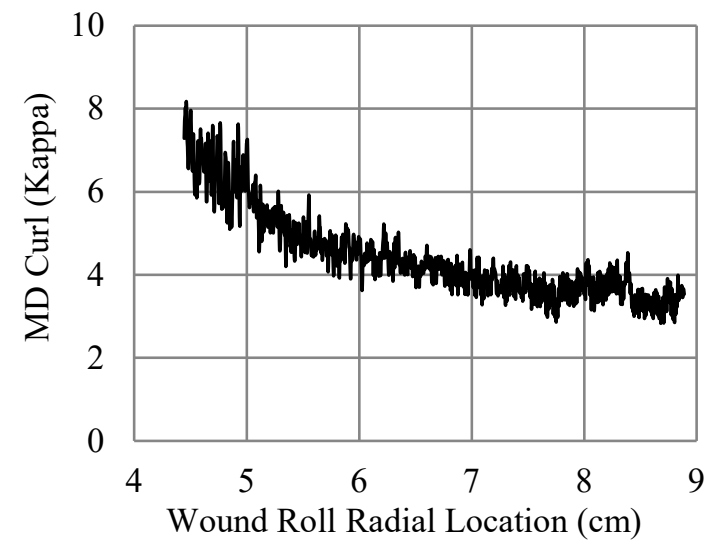

Figure 14 - Online MD Curl after 1Day Storage (Kappa Unit)

After several reversed windings with intermediate storage, the online measurement in Figure 13 shows the majority of the wound roll has initial curl less than 2 Kappa units. The roll was then stored one day at room temperature $\left(21^{\circ} \mathrm{C}\right)$ and the online measurement was conducted again. The MD curl radius as inferred from online measurement as a function of radial storage location in the wound roll is shown in Kappa units in Figure 14. The radius of MD curl inferred from online measurement and the storage location in the wound roll for the layer(s) that contributed to those measurements is shown for the same roll in Figure 15. Charts of bending recovery versus radial storage location could be developed using this data. 


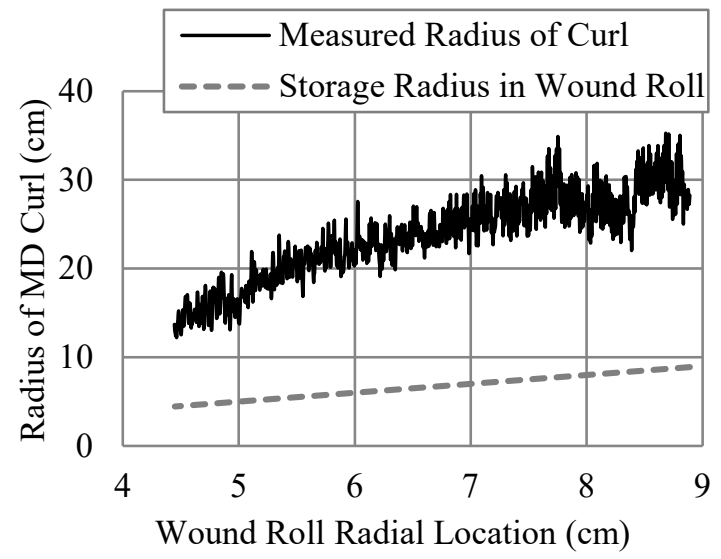

Figure 15 - Online MD Curl after 1Day Storage

Earlier in the validation effort for the MD curl software, specimens of web were cut from the innermost and outermost layer to directly measure the MD curl using the Kappa Gauge. Those results compared very well with the online measurements in Table 7.

\begin{tabular}{|l|c|c|}
\hline & $\begin{array}{l}\text { Kappa Gage MD Curl } \\
\text { Measurement }\end{array}$ & $\begin{array}{l}\text { Online MD Curl } \\
\text { Measurement }\end{array}$ \\
\hline Innermost Layer & $8.0 \mathrm{Kappa}$ & $7.7 \mathrm{Kappa}$ \\
\hline Outermost layer & $3.5 \mathrm{Kappa}$ & $3.8 \mathrm{Kappa}$ \\
\hline
\end{tabular}

Table 7 - Comparison of Kappa Gage and Online MD Curl Measurement

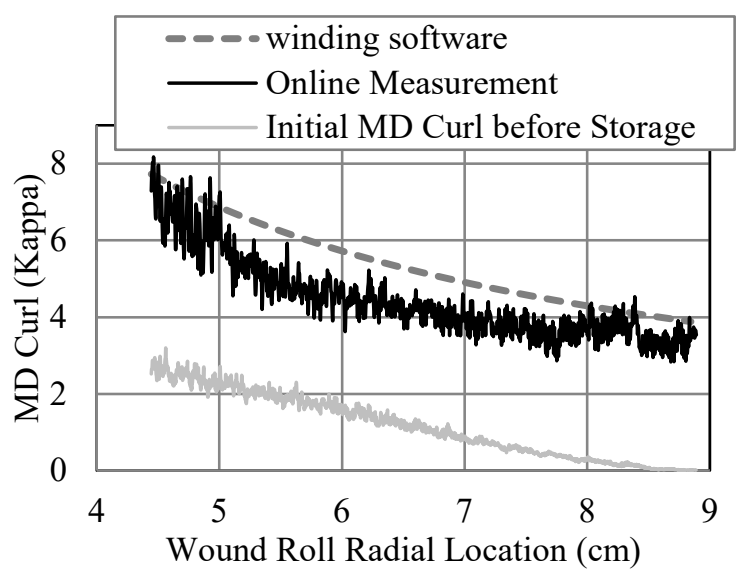

Figure 16 - Comparison between Online Measurement and winding software results.

A comparison between the online measurement and the winding software results is shown in Figure 16. For the outermost layer and innermost layer, the results compare well. The error is greater for the interior of the wound roll. While it is possible that dissimilar relaxation $E_{c}\left(t_{r}\right) \neq E_{t}\left(t_{r}\right)$ may cause this error in the interior region, the 
compressive relaxation modulus was not characterized, and this error due to this simplification cannot be estimated. Friction will affect slippage and the location of the neutral axis and hence the portions of the web that experience tension and compressive stress. This could further amplify the effects of dissimilar relaxation.

\section{CONCLUSIONS}

Methods were explored to predict MD curl in wound rolls due to web viscoelasticity. The developed methods included a winding software, dynamic simulations, and an online direct measurement. The analysis methods and the online measurement method all demonstrated the ability to predict and/or measure the MD curl defect. The curl in an anisotropic web and the effect of dissimilarities between tensile and compressive relaxation functions remain the subject of future work. The predictive methods could potentially be used to limit storage times and temperatures in order to mitigate MD curl. The online measurement method is useful for quantifying MD curl in webs stored in wound rolls for indefinite periods of time or for cases where reversed windings and intermediate storage is being used to eliminate MD curl.

\section{ACKNOWLEDGEMENTS}

The authors would like to thank the sponsors of the Web Handling Research Center for providing the support which made this research possible.

\section{REFERENCES}

1. Hakiel, Z., "Nonlinear Model for Wound Roll Stresses," TAPPI J., Vol. 70, No. 5, May 1987, pp. 113-117.

2. Mollamahmutoglu, C., and Good, J. K., "Analysis of Large Deformation Wound Roll Models," ASME Journal of Applied Mechanics, Vol. 80, July 2013, pp. 041016-1-11.

3. Qualls, W. R., "Hygrothermomechanical Characterization of Viscoelastic Centerwound Rolls," PhD thesis, Oklahoma State University, 1995.

4. Qualls, W.R., and Good, J.K., "Viscoelasticity in Wound Rolls," ASME J. Appl. Mech., Vol. 64, No. 1, March 1997, pp. 201-208.

5. Smith, R.D., Roll and Web Defect Terminology, $2^{\text {nd }}$ edition, TAPPI Press, 2007.

6. Timoshenko, S., and Woinowsky-Kreiger, S., Theory of Plates and Shells, 2nd ed. McGraw-Hill, New York, 1959, pp. 33-44.

7. Schrader, W., and Carroll, J. F., "Process for Reducing Core-Set Curling Tendency and Core-Set Curl of Polymeric Films," US41417345A, 1979.

8. Maier, K. L., Moszkowicz, J. M., and Laney, M. T., "Process for Controlling Curl in Polyester Film," US5076977A, 1991.

9. Onishi, T., and Dotani, S., "Process for Controlling Curl in Coated Papers," US4853255A, 1989.

10. Greener, J., Tsou, A. H., Ng, K. C., and Chen, W. A., "The Bending Recovery of Polymer Films," Journal of Polymer Science, Part B, Polymer Physics, 1991.

11. Greener, J., and Gillmor, J. R., "Long-Term Growth of Core-Set Curl in Poly(Ethylene Terephthalate) Film," Journal of Polymer Science, Part B, Polymer Physics, 2001. 
12. Kidane, S., "Laminate Theory Based 2D Curl Model," Proceedings of the Tenth International Conference on Web Handling, Web Handling Research Center, Oklahoma State University, Stillwater, Oklahoma, 2009, pp. 387-401.

13. Swanson, R. P., "Web Curl and Web Curl Measurement," Proceedings of the 14th International Conference on Web Handling, Web Handling Research Center, Oklahoma State University, Stillwater, Oklahoma, 2017.

14. Matsuoka, S., Failure of Plastics, W. Brostow and R. D.Corneliussen, eds., Hanser Publishers, New York, 1987, p. 24.

15. Pfeiffer, J. D., "Internal Pressures in a Wound Roll of Paper," TAPPI Journal, Vol. 49, No. 8, 1966, pp. 342-347.

16. Baumgaertel, M., and Winter, H. H., "Determination of Discrete Relaxation and Retardation Time Spectra from Dynamic Mechanical Data," Rheologica Acta, 28, pp. 511-519, 1989. 


\section{APPENDIX}

\begin{tabular}{|c|c|}
\hline Web thickness & $0.0508 \mathrm{~cm}$ \\
\hline Width & $10.16 \mathrm{~cm}$ \\
\hline CMD Modulus & $153.06 \mathrm{MPa}$ \\
\hline MD Modulus & $153.06 \mathrm{MPa}$ \\
\hline
\end{tabular}

Table A1 - LDPE properties for winding software $\left(21^{\circ} \mathrm{C}\right)$

\begin{tabular}{|c|c|}
\hline Core OD & $8.89 \mathrm{~cm}$ \\
\hline Core ID & $7.62 \mathrm{~cm}$ \\
\hline Material Modulus & $6.8 \mathrm{E} 5 \mathrm{MPa}$ \\
\hline Calculated Core Stiffness & $1.1 \mathrm{E} 5 \mathrm{MPa}$ \\
\hline Poisson's Ratio of Core & 0.3 \\
\hline Wound Roll OD $(\mathrm{cm})$ & 24.1 (test 1$), 22.9$ (test 2$), 22.1$ (test3), \\
& $21.8($ tests 4,5$)$ \\
\hline Winding Tension $(\mathrm{KPa})$ & $775($ tests1,2,3), 1550(test4), 430(test5) \\
\hline $\mathrm{K} 1(\mathrm{KPa})$ & $6.9 \mathrm{E}-05$ \\
\hline $\mathrm{K} 2$ & 246.5 \\
\hline
\end{tabular}

Table A2 - Input parameters winding software

\begin{tabular}{|c|c|}
\hline \multicolumn{2}{|c|}{ MD Creep Terms } \\
\hline $\mathrm{J}_{1}$ & $-2.9 \mathrm{E}-06 \mathrm{~m} / \mathrm{m} / \mathrm{KPa}$ \\
\hline $\mathrm{J}_{2}$ & $-1.16 \mathrm{E}-06 \mathrm{~m} / \mathrm{m} / \mathrm{KPa}$ \\
\hline$\tau_{1}$ & $1913 \mathrm{~s}$ \\
\hline$\tau_{2}$ & $100,566 \mathrm{~s}$ \\
\hline \multicolumn{2}{|c|}{ Radial Creep Terms } \\
\hline $\mathrm{J}_{1}$ & $-1.29 \mathrm{E}-06 \mathrm{~m} / \mathrm{m} / \mathrm{KPa}$ \\
\hline $\mathrm{J}_{2}$ & $-1.35 \mathrm{E}-06 \mathrm{~m} / \mathrm{m} / \mathrm{KPa}$ \\
\hline$\tau_{1}$ & $696 \mathrm{~s}$ \\
\hline$\tau_{2}$ & $72,810 \mathrm{~s}$ \\
\hline
\end{tabular}

Table A3 - Viscoelastic input for winding software $\left(21^{\circ} \mathrm{C}\right)$ 